\title{
TOPODIAGNÓSTICO NA PARALISIA FACIAL PERIFÉRICA
}

\author{
RICARDO E. BENTO * \\ EDUARDO A. B. VELLUTINI*** \\ FELIX H. PAHL *** \\ A. J. TEDESCO-MARCHESE** \\ GILBERTO S. FORMIGONI**** \\ HECTOR C. NAVARRO*** \\ A. MINITI*
}

O topodiagnóstico na paralisia facial tem como objetivo a loçalização anatômica precisa da lesão neural para fornecer maiores subsidios a eventual exploração cirúrgica e para fornecer dados sobre o prognóstico e evolução do quadro. Clinicamente, deve ser determinado se a lesão é periférica ou central. Nesta última, devido às conexões bilaterais da porção do núcleo do facial responsável pela musculatura frontal, não encontramos déficit motor em andar superior. Por outro lado, pacientes com lesão do núcleo ou do nervo facial propriamente dito apresentam déficit em toda a hemiface comprometida. $\mathrm{Na}$ lesão dita periférica do facial, podemos precisar o local de comprometimento do nervo testando a função de cada um de seus ramos 1 . O primeiro ramo é o nervo petroso superficial maior, com emergência na altura do gânglio geniculado, responsável pela inervação da glândula lacrimal homolateral. $\mathrm{O}$ segundo, ramo do estapédio, controla a contração do músculo estapédio no ouvido interno. O terceiro, nervo corda do tímpano, é responsável pela função gustativa dos 2/8 anteriores da língua e pela secreção da glândula salivar submandibular homolateral (Fig. 1).

O Grupo de Paralisia Facial do Hospital das Clínicas da FMUSP utiliza a seguinte rotina diagnóstica para testar individualmente cada um desses ramos: 1. Teste de lacrimejamento ou de Schirmer - Consiste na análise quantitativa e comparativa da função da glândula lacrimal. A quantidade de secreção lacrimal pode ser medida diretamente através de tiras de papel de filtro de $0,5 \mathrm{~cm}$ de largura por $5 \mathrm{~cm}$ de comprimento, que são colocadas no terço médio da pálpebra inferior após ter sido cuidadosamente seca, tomando-se a precaução de não irritar a conjuntiva. Após um minuto, é efetuada a medição, sendo consideradas patológicas diferenças superiores a 1/3. Pode-se ainda realizar a prova com estímulo olfatório de amônia 4. Considerando-se o teste patológico, suspeita-se de lesão supra-geniculada. 2. Reflexo do músculo estapédio - $\mathrm{O}$ músculo estapédio tem a função de proteger o ouvido interno de sons de alta inten-

Trabalho da Divisão de Neurologia e da Divisão de Otorrinolaringologia do Hospital das Clínicas da Faculdade de Medicina da Universidade de são Paulo (FMUSP): * Médico Assistente, Otorrinolaringologia; ** Médico Assistente, Neurologia; *** Médico Residente, Neurologia; *** Médico Residente, Otorrinolaringologia. 


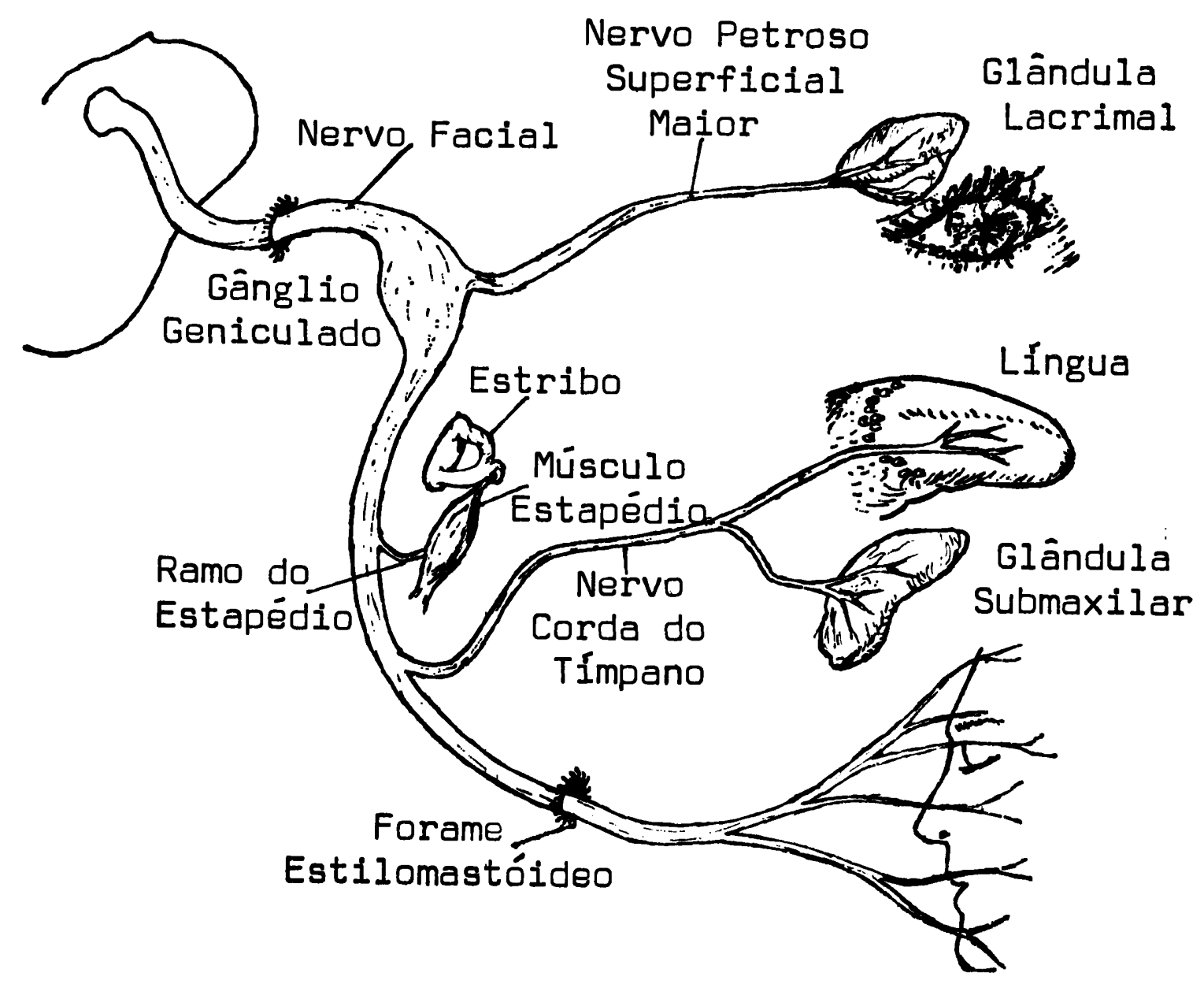

Fig. 1 - Distribuiço dos ramos do nervo facial; esquema baseado no de Alford e col.1.

sidade, contraindo-se e enrijecendo a cadeia ossicular, aumentando assim a impedância acústica do mecanismo de transmissão sonora. Em pessoas normais, tons puros de aproximadamente 85 decibéis são suficientes para causar esta contração. O reflexo de contração é bilateral e simultâneo. Para realizarmos este teste, utilizamos o impedanciômetro, que irá medir a contração do estapédio no lado afetado, após estímulo sonoro no ouvido são. Para a realização do teste, é necessário que o paciente apresente função auditiva e estrutura do ouvido preservadas. Quando não se observa a contração do estapédio, suspeitamos de lesão acima da emergência desses ramos (supraestapediana). 3. Terceiro ramo do facial - Conhecido como nervo corda do tímpano, é responsável pela gustação nos $2 / 3$ anteriores da língua e pela secreção salivar submandibular homolateral. A gustometria química é por nós usada para testar a função deste ramo. Este teste consiste na colocação de três tipos de sabores (doce, azedo e amargo) com cotonete nos $2 / 3$ anteriores da língua e solicitar ao paciente que aponte em um cartaz o sabor que está sentindo. Não utilizamos de rotina o teste para volume de salivação, pois seus resultados na prática são discutíveis. Não obtivemos bons resultados com a eletrogustometria em nossos pacientes. Se a função do nervo corda do tímpano estiver alterada, suspeitamos de uma lesão supracordal. Se os três ramos estiverem normais, a lesão é dita infracordal.

Mediante a utilização desses três testes de fácil execução, podemos suspeitar do local de comprometimento do facial para melhor planejamento cirúrgico. 
É propósito deste estudo apresentar resultados pertinentes a 873 casos de paralisia facial periférica.

\section{CASUISTICA E RESULTADOS}

De 1978 até 1983 foram atendidos no ambulatório do Grupo de Paralisia Facial do Hospital das Clínicas da FMUSP 873 pacientes. O topodiagnóstico obedeceu aos princípios descritos. Todos eles foram submetidos a exames gerais no intuito de identificar fatores etiológicos. Além disso, foram realizadas audiometria e planigrafia de ouvido para estudo do canal do facial. Após esses testes os pacientes foram classificados em grupos de acordo com a localização e a etiologia (Tabelas 1, 2 e 3).

O principal grupo é representado pelas paralisias faciais ditas idiopáticas (289 casos: $33,2 \%$ ) sendo $54 \%$ delas suprageniculadas. Seguem-se as de etiologia traumática (273 casos: 31,3\%) sendo também representadas na sua maioria pelas suprageniculadas (51,3\%). Destacam-se neste grupo as lesões de localização infrageniculadas e supracordais

\begin{tabular}{lrr}
\hline & $\begin{array}{c}\text { Número de } \\
\text { casos }\end{array}$ & $\%$ \\
\hline \hline Suprageniculada & 430 & 49,26 \\
Infrageniculada, supraestapediana & 97 & 11,11 \\
Infraestapediana, supracordal & 67 & 7,67 \\
Infracordal & 133 & 15,23 \\
Infrageniculada, supracordal & 106 & 12,15 \\
Duvidoso & 40 & 4,58 \\
\hline
\end{tabular}

Tabela 1 - Topadiagnóstico nos 873 casos de paralisia facial periferica.

\begin{tabular}{lcc}
\hline & $\begin{array}{c}\text { Número de } \\
\text { casos }\end{array}$ & $\%$ \\
\hline \hline Idiopática & 289 & 33,2 \\
Traumática & 273 & 31,3 \\
Infecciosa & 97 & 11,1 \\
Metabólica & 84 & 9,6 \\
Herpética & 49 & 5,6 \\
Tumoral & 32 & 3,6 \\
Tóxica & 12 & 1,4 \\
\hline
\end{tabular}

Tabela 2 - Distribuição dos 873 casos de paralisia facial periferica segundo fossem idiopdtiaas ou relacionadas a determinados fatores etiologicos. 


\begin{tabular}{|c|c|c|c|}
\hline & Topodiagnóstico & $\begin{array}{l}\text { Número de } \\
\text { casos }\end{array}$ & $\%$ \\
\hline \multirow[t]{5}{*}{ Idiopática } & Suprageniculada & 156 & 54 \\
\hline & Infrageniculada, supraestapediana & 72 & 25 \\
\hline & Infraestapediana, supracordal & 53 & 19 \\
\hline & Infracordal & 4 & 1 \\
\hline & Duvidoso & 4 & 1 \\
\hline \multirow[t]{6}{*}{ Traumática } & Suprageniculada & 140 & 51,3 \\
\hline & Infrageniculada, supraestapediana & 5 & 1,9 \\
\hline & Infraestapediana, supracordal & 2 & 0,7 \\
\hline & Infracordal & 106 & 38,8 \\
\hline & Infrageniculada, supracordal & 17 & 6,2 \\
\hline & Duvidoso & 3 & 1,1 \\
\hline \multirow[t]{3}{*}{ Infecciosa } & Suprageniculada & 6 & 6,2 \\
\hline & Infrageniculada, supracordal & 87 & 89,7 \\
\hline & Infracordal & 4 & 4,1 \\
\hline \multirow[t]{5}{*}{ Metabólica } & Suprageniculada & 48 & 57,1 \\
\hline & Infrageniculada, supraestapediana & 20 & 23,8 \\
\hline & Infraestapediana, supracordal & 11 & 13,1 \\
\hline & Infracordal & 0 & 0 \\
\hline & Duvidoso & $\mathbf{5}$ & 6,0 \\
\hline Herpética & Suprageniculada & 49 & 100 \\
\hline \multirow[t]{5}{*}{ Tumoral } & Suprageniculada & 17 & 53,1 \\
\hline & Infrageniculada, supraestapediana & 0 & 0 \\
\hline & Infraestapediana, supracordal & 1 & 3,1 \\
\hline & Infracordal & 14 & 43,8 \\
\hline & Duvidoso & 0 & 0 \\
\hline Tóxica & Suprageniculada & 12 & 100 \\
\hline
\end{tabular}

Tabela 3 - Topodiagnóstico dos 873 casos de paralisia facial periférica distribuídos segundo fossem idiopáticos ou relacionados a determinados fatores etiológicos.

que representaram lesões extensas atingindo o nervo facial em quase toda sua extensão no trajeto ósseo.

Naqueles pacientes em que foi possivel correlacionar a paralisia a uma causa infecciosa (11,1\%), predominaram as lesões extensas (infrageniculada-supracordal: 89,7\%) causadas principalmente por infecções do ouvido médio e parotidites. No restante dos pacientes com paralisia facial de origem metabólica $(9,6 \%)$, herpética $(5,6 \%)$, tumoral $(3,6 \%)$ e tóxica $(1,4 \%)$, também predominaram as lesões suprageniculadas, que no total geral somaram praticamente $50 \%$ de todos pacientes. 


\section{COMENTARIOS}

Em concordância aos dados da literatura 3 , observa-se na estatística apresentada que a lesão suprageniculada é a mais frequente, tanto no grupo de paralisias faciais ditas idiopáticas, com o maior número de casos, como nas paralisias faciais de etiologia conhecida (traumática, tumoral, metabólica, herpética, tóxica).

$O$ tipo de tratamento dos pacientes com paralisia facial periférica irá depender da etiologia, quadro clínico e estudo eletrofisiológico do nervo. Se o tratamento cirúrgico for o indicado, o topodiagnóstico é importante na determinação da porção do nervo a ser explorada 2.

A via de acesso utilizada para a abordagem da primeira porção do facial (suprageniculado) nos pacientes com audição preservada é a fossa média, isto é, abordagem do conduto auditivo interno através de craniotomia temporal baixa com visualização do assoalho da fossa média, para a exploração do nervo facial. As lesões suprageniculadas sem preservação da audição do mesmo lado, podem ser abordadas por via translabiríntica. As lesões infrageniculadas e supracordais são atingidas pela via transmastóidea, visualizando-se a segunda e a terceira porções do nervo através de mastoidectomia. Nas lesões infracordais, isto é, após ou próximo à emergência do facial no forame estilomastóideo, a via utilizada é a pré-auricular.

A localização exata da lesão através do topodiagnóstico é elemento fundamental ao se optar por exploração cirúrgica do nervo facial e deve ser feita o mais precocemente possível, para que se evitem falsos resultados, pois estes testes tendem a se normalizar após período variável de paralisia.

\section{RESUMO}

A importância do topodiagnóstico na paralisia facial periférica é a localização anatômica precisa da lesão neural. Consiste ele na realização de testes clínicos para avaliar as funções de cada um dos ramos do nervo. O Grupo de Paralisia Facial do Hospital das Clínicas da Faculdade de Medicina da Universidade de São Paulo, com estatística de 873 pacientes, demonstra que praticamente $50 \%$ dos casos, das mais diversas etiologias, apresentavam lesão suprageniculada. A importância desse dado é a indicação da via de acesso, quando necessária a exploração cirúrgica do nervo facial.

\section{SUMMARY}

\section{Topodiagnostics of peripheral facial palsies.}

The topodiagnosis allows the physician to determine the topographic localization of pathology involving the facial nerve. It is based on clinical tests 
that evaluate the function of each one of the rami of the facial nerve. With a statistic of 873 patients, the Facial Nerve Group of Hospital das Clinicas, São Paulo University, found that suprageniculate lesions are responsible for $50 \%$ of the facial nerve involvement of several etiologies. Based on these results, the physician is able to develop a rationale for therapy and surgical access for facial nerve lesions.

\section{REFER ENCIAS}

1. ALFORD, B.; JERGER, J.F.; COATS, A.C.; PETERSON, C.R. \& WEBER, J.R. Neurophysiology of facial nerve testing. Arch. Otolaryngol. 97:214, 1973.

2. FISCH, U. - Surgery for Bell's palsy. Arch. Otolaryngol. 107:1, 1981.

3. FISCH, U. \& FELIX, H. - On the pathogenesis of Bell's palsy. Acta Otolaryngol. $95: 532,1983$.

4. GONTRER, J. \& FISCH, U. - Schirmer's test: its normal values and clinical significance. ORL 38:1, 1976.

Grupo de Paralisia Facial, Hospital das Clínicas, FMUSP - Caixa Postal 3461 - 01000 , são Paulo, SP - Brasil. 